\title{
Belphégor
}

\section{Série ou collection? Notes sur le roman-photo}

\author{
Jan Baetens
}

\section{(2) OpenEdition}

\section{Journals}

\section{Édition électronique}

URL : https://journals.openedition.org/belphegor/3923

DOI : 10.4000/belphegor.3923

ISSN : 1499-7185

Éditeur

LPCM

\section{Référence électronique}

Jan Baetens, "Série ou collection? Notes sur le roman-photo », Belphégor [En ligne], 19-1 | 2021, mis en ligne le 24 juin 2021, consulté le 06 juillet 2021. URL : http://journals.openedition.org/belphegor/ 3923 ; DOI : https://doi.org/10.4000/belphegor.3923

Ce document a été généré automatiquement le 6 juillet 2021.

\section{(c) (i) (9)}

Belphégor est mis à disposition selon les termes de la Licence Creative Commons Attribution - Pas d'Utilisation Commerciale - Pas de Modification 4.0 International. 


\title{
Série ou collection? Notes sur le roman-photo
}

\author{
Jan Baetens
}

1 Série et collection sont des amis, mais de faux amis. De l'une à l'autre, les correspondances sont nombreuses, mais pas au point d'en faire de vrais synonymes (cela devient tout à fait patent dès qu'on passe du substantif au verbe : sérialiser dénote clairement une activité autre et surtout moins large que collectionner). Collection et série se touchent, se chevauchent, se superposent, sans pour autant coïncider. Objet sériel en même temps qu'objet de collection, le roman-photo offre une belle occasion de réfléchir aux ressemblances et dissymétries des deux concepts et des deux pratiques, en l'occurrence du point de vue de leur genèse. Dans le contexte du roman-photo, la production de la série et celle de la collection obéissent à des logiques différentes, dont il faut espérer que l'écart apportera quelque chose à l'étude du travail en série en général.

\section{Une sérialité faite de multiples séries}

Vu de loin, il n'y a aucune raison d'aller au-delà du cadre de la série pour parler du roman-photo. Celui-ci combine tous les traits distinctifs d'une production sérielle au sein de l'industrie culturelle du livre, plus exactement de l'industrie des magazines féminins comme on la voit réapparaître pour se réinventer après la Seconde Guerre mondiale (Giet 1998, Antonutti 2013, Baetens 2017). Quand il surgit le 8 mai 1947, dans le premier numéro de Il Mio Sogno («Mon Rêve»), rapidement rebaptisé en Sogno ("Rêve »), magazine qui s'autoproclame en bandeau : "settimanale di romanzi d'amore a fotogrammi » (littéralement : « hebdomadaire de romans d'amour à photographies »), la sérialité est inscrite dans l'ADN du nouveau genre. On peut examiner cette dimension sérielle à partir de trois perspectives : textuelle (interne), hypertextuelle (c'est ici qu'on pourrait situer le moment proprement génétique du roman-photo en tant que genre ${ }^{1}$ ) et intertextuelle ou transmédiatique (soit culturelle au sens très large du terme). 
Détaillons d'abord quelques aspects de la sérialité de l'objet même. Sogno, puis un nombre vite croissant de périodiques comparables copient sans exception la même formule, dont le caractère répétitif est mis en lumière par de nombreux témoignages visuels de l'époque. Le kiosque affichant des dizaines de magazines tous pareils est un motif favori du genre, qui s'en sert volontiers dans sa propre publicité, à l'intérieur ou à l'extérieur des publications. Plus violent encore est le pêle-mêle qu'on trouve en quatrième de couverture d'un numéro spécial de Regards (Fig. 1), mensuel culturel lié au PCF qui avait rejoint la grande campagne anti-roman-photo, la presse du cœur alors synonyme de roman-photo- étant accusée de "démoraliser», c'est-à-dire d'embourgeoiser, ses lectrices (on passera vite sur le constat établi par toutes les enquêtes sociologiques qu'un pourcentage tout sauf symbolique de ces lectrices étaient en fait des lecteurs, voir Sullerot 1963 et Bravo 2003 ; sur l'échec de cette croisade antiphoto-romanesque, menée de front par la gauche et la droite, voir Bonifazio 2017) :

Fig. $1: 4^{\mathrm{e}}$ de couverture de Regards, $\mathrm{n}^{\circ} 352$, août 1952 ("Les Secrets de la presse du cœur »). On reconnaît, de gauche à droite et de haut en bas : Rêves, Intimité, Madrigal, Confidences, Ėve, Festival, Nous Deux, Boléro, Intimité et $A$ tout cœur)

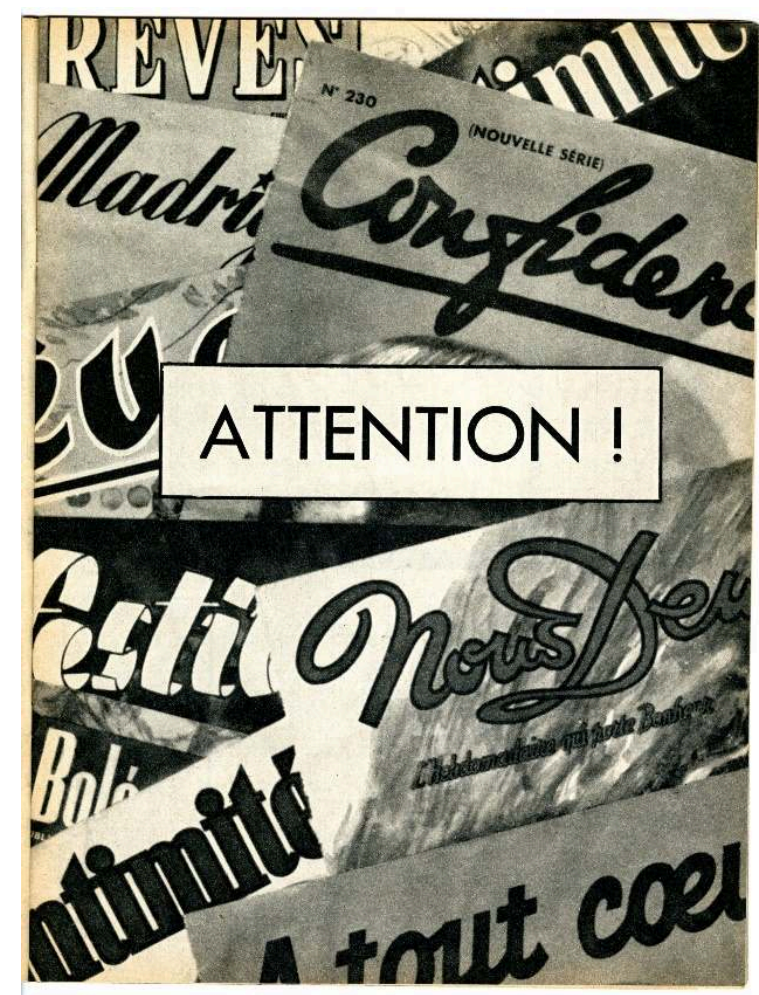

Directement liées à la sérialité, les contraintes régissant le roman-photo des origines peuvent se décrire comme suit : 1) conception et réalisation de l'œuvre sous forme de feuilleton, non de récit complet : cette fragmentation cherche à fidéliser le lectorat, peu enclin à s'abonner aux revues, car autant le prix au numéro était très abordable, autant le prix de l'abonnement représentait toujours un réel écueil; 2) cadence de production élevée: les feuilletons se publiaient à raison de quelques pages par semaine, généralement de trois à six, et sauter une semaine n'était pas une option (il ne faut pas oublier que les romans-photos étaient livrés au rythme même de leur production : on pouvait certes prendre un peu d'avance, mais comme le succès éventuel d'un feuilleton 
entraînait souvent son allongement de manière à l'exploiter au maximum, il n'était pas toujours possible de l'avoir terminé au moment du lancement) ; 3) conformité absolue aux règles narratives et stylistiques d'une certaine littérature, celle du mélodrame hérité des romans $\mathrm{du} 19^{\mathrm{e}}$ siècle, et conformité non moins inconditionnelle à la «ligne » ou à la tonalité spécifique de chaque magazine, toutes les publications étant sans arrêt à la recherche de ce qui leur permettait à la fois de ressembler parfaitement au modèle implicite du genre et de se distinguer par quelques détails plus ou moins importants (les romans-photos belges, par exemple, se vantaient d'offrir une lecture "pour tous ", à la différence de la production française jugée un tantinet impudique ; corollairement, la Belgique se montrait aussi plus ouverte à l'ironie et au sourire, sans jamais tomber, disait-on, dans les excès typiquement français du "bête et méchant »);4) déséquilibre radical entre éditeur et auteur : celui-ci souvent anonyme et réduit à une fonction de simple exécutant, celui-là porte-parole de l'instance hégémonique du roman-photo : le véritable auteur d'un roman-photo n'est ni le photographe, ni le scénariste, ni le metteur en pages, ni l'acteur, mais le magazine qui passe commande de l'œuvre.

Ces contraintes, le roman-photo les invente d'autant moins qu'il les reprend intégralement à un autre genre alors très en vogue dont il se présente comme la continuation directe, pour ne pas dire l'exacte mais plus efficace copie -confusion volontaire ou involontaire qu'on voit très bien dans le documentaire de Michelangelo Antonioni, L'amorosa menzogna (1949, « Le mensonge amoureux »), bref reportage sur la production, la distribution et enfin la lecture du roman-photo éponyme où le directeur mélange de façon indistincte le roman-photo et le genre qu'il imite. Cet ancêtre hypertextuel est le "roman dessiné ", né dans la même presse du cœur à peine un an plus tôt, le 27 juin 1946 dans les colonnes de l'hebdomadaire Grand Hôtel (transposé et adapté un an plus tard en France sous le titre de Nous Deux). La clé du succès aussi soudain que colossal de Grand Hôtel, puis de Nous Deux, tient entièrement à l'invention de ce roman dessiné, qui combine avec brio trois grandes veines de la culture populaire de l'immédiat après-guerre : le mélodrame, la bande dessinée, le cinéma (hollywoodien plutôt que néo-réaliste). Un roman dessiné se présente comme une sorte de « romance comic » (avant même que ce type de bande dessinée naisse aux États-Unis, en 1948, il est vrai dans un contexte tout autre, celui des comic books, non celui de la presse du cœur), exécuté dans un style photoréaliste et plagiant sans le moindre état d'âme les situations narratives et surtout le physique des vedettes du grand écran. Le résultat de l'opération est à la fois visuellement éblouissant et subtil du point de vue narratif (Fig. 2), mais difficile à maintenir dès que la formule commence à se répandre. 
Fig. 2 : «Âmes ensorcelées ", page inaugurale du premier roman dessiné dans Nous Deux, 14 mai 1947 (il s'agit d'une traduction/adaptation de la version italienne parue d'abord dans Grand Hôtel sous le titre de " Anime incatenate»)

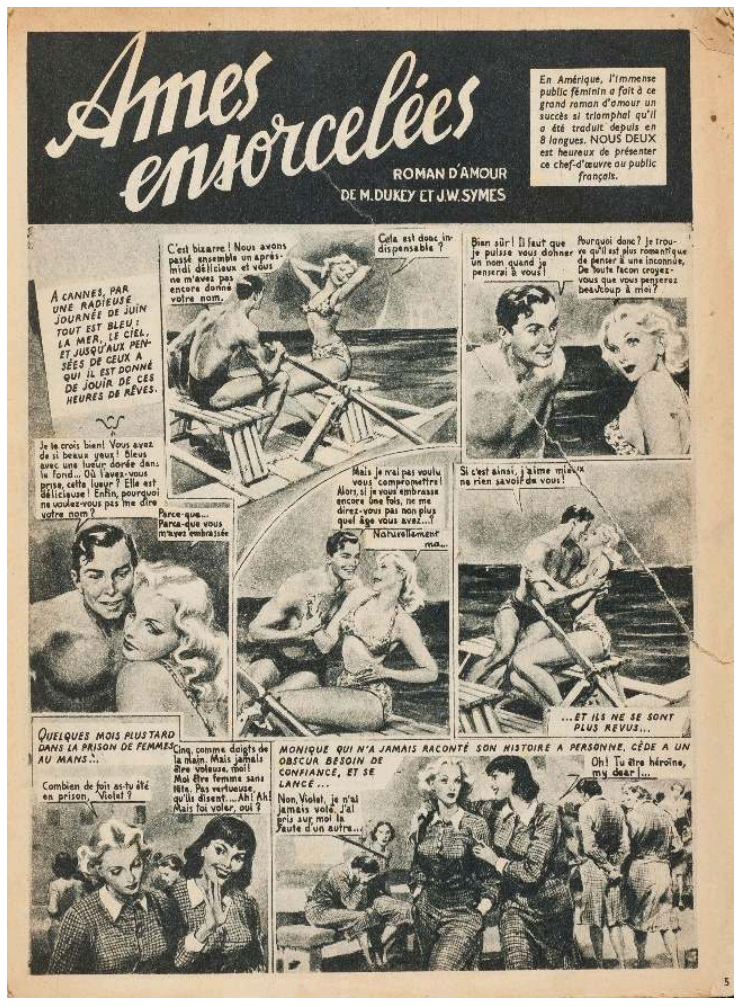

6 En effet, les artistes capables de fournir des planches de même qualité qu'Âmes ensorcelées, modèle inégalé du genre, sont rares et plus rares encore sont ceux capables de tenir aussi le rythme effréné imposé par la concurrence entre les magazines avides d'inclure autant de pages de roman dessiné que possible. La solution trouvée est d'une simplicité biblique: on remplacera les dessins par des photos (je souligne le pronom impersonnel « on », car l'origine précise du nouveau genre reste sujette à controverse, voir Faber, Minuit et Takodjerad 2012: 39). Or, le passage du roman dessiné au romanphoto crée autant de problèmes qu'il n'en résout. Le nouveau genre a beau régler la question économique qui torturait les éditeurs, il brille aussi par sa relative pauvreté esthétique. Les budgets manquent pour construire de vrais décors. Les acteurs et actrices sont loin d'être toujours aussi sexy que les stars d'Hollywood qu'on se faisait un plaisir de retrouver dans le roman dessiné. L'intégration des mots et des images est souvent maladroite. Le rythme narratif s'enlise. Enfin l'impression des clichés photographiques souffre beaucoup de la mauvaise qualité du papier. Les difficultés de la transition se notent surtout dans la maladresse des tentatives d'aligner les premiers romans-photos sur les propriétés de son prédécesseur, très visibles dans le recadrage des images et leur mise en pages "expressive " (c'est-à-dire irrégulière, aux angles brisés ou arrondis), mais aussi dans le recours systématique à la retouche, voire au coloriage. Manœuvres de camouflage vite abandonnés, car plus ridicules qu'efficaces, et $\mathrm{du}$ reste peu conformes aux souhaits du public, moins désireux de se projeter dans l'usine à rêves que de se reconnaître dans les images photographiques de la femme ou de l'homme d'à côté (femme et homme plutôt que garçon et fille, le lectorat cible étant transgénérationnel). 
7 En troisième lieu, et je passe ici à la dimension intertextuelle et transmédiatique de la genèse du roman-photo, il faut noter que le nouveau genre se trouve à la croisée d'un grand nombre d'autres séries médiatiques, dont avant tout du cinéma. Venant du monde et de l'imaginaire $\mathrm{du}$ film, par le biais des personnages et intrigues du roman dessiné, le roman-photo sert aussi de courroie de transmission vers le cinéma, quand bien même son propre langage est trop démuni et indigent pour se faire accepter comme double d'une fiction hollywoodienne. D'un côté, le roman-photo, rapide et peu coûteux à réaliser, se promeut comme banc d'essai pour les sujets qui, en cas de succès commercial, pourront être explorés, voire exploités, c'est-à-dire repris en série, à Cinecittà ou ailleurs. De l'autre, il représente aussi un tremplin pour une carrière au cinéma, tout à coup ouverte au grand public grâce aux castings délocalisés où les magazines cherchent à recruter leurs nouvelles vedettes. Sogno le fera dès son premier numéro et tel fut aussi le cursus honorum de certaines stars ou dive, sautant du concours de beauté au roman-photo et de là au tournage d'un premier film.

\section{... mais en manque de collection}

Or tous ces aspects, internes comme externes, qui jouent un rôle capital dans l'émergence et plus tard la consolidation du roman-photo, ont un point en commun: aucun d'eux n'aura été à même de transformer l'effet de série en véritable collection. Le romanphoto est incontestablement partout, sauf dans de véritables collections à lui. Mais que signifie cette absence?

9 Les Éditions Mondiales, leader de marché pour la presse du cœur, lance en 1948 une collection spéciale «Les romans dessinés de Nous Deux », dont le premier volume sera -évidemment ! - Âmes ensorcelées (Fig. 3). 
Fig. 3 : M. Dukey et J. W. Symes, Âmes ensorcelées. Paris : Éd. Nous Deux, 1948 (à noter que l'œuvre, sans nom d'auteur en première de couverture, est attribuée en page de faux titre à deux auteurs fictifs, soi-disant américains ; l'édition originale italienne était même présentée comme une traduction de l'américain -on se croirait chez Boris Vian !)

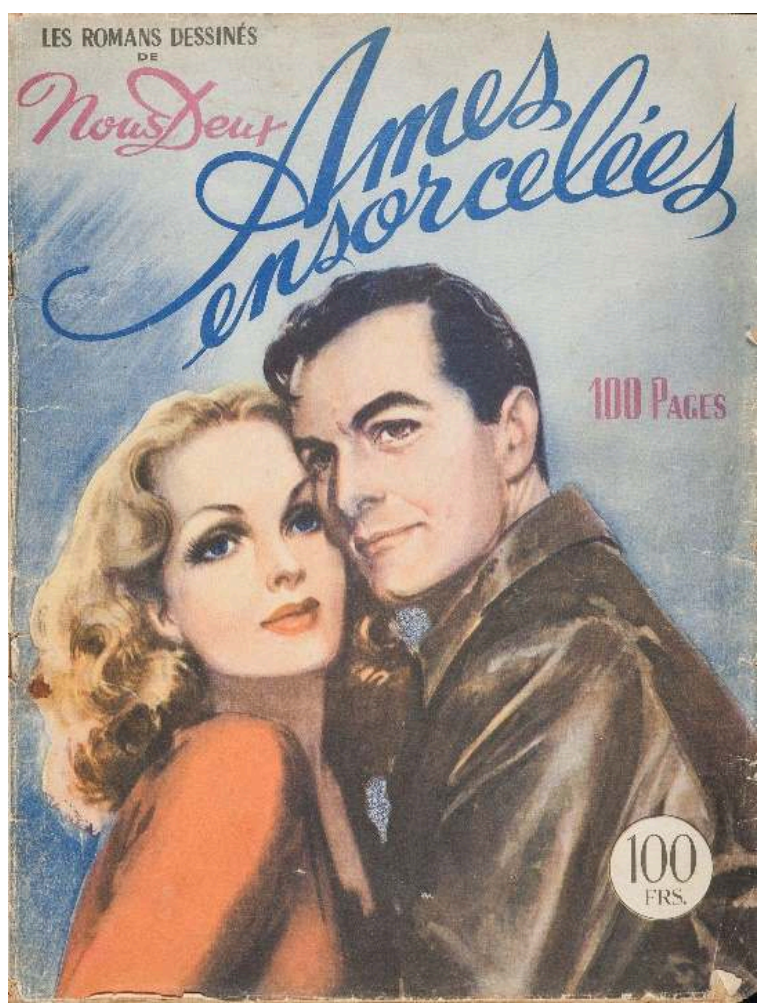

Pareille publication semble renouer avec une tradition bien connue : la reprise d'un feuilleton à grand succès sous forme de livre (dans le cas qui nous concerne ici, l'œuvre était sortie en trente épisodes hebdomadaires). Mais les apparences sont ici partiellement trompeuses. Du point de vue matériel, on reste proche du format d'origine: les numéros de la collection ont mêmes dimensions, même papier, même agrafage, même séparation du noir et blanc (à l'intérieur) et de la couleur (en couverture), enfin même type de couverture (d'une épaisseur égale à celles des pages d'intérieur). Ce qui change par contre, c'est le prix : $100 \mathrm{~F}$ (à peu près 3,50 euros), là où un numéro simple de Nous Deux de la même période (novembre 1948) ne coûte que $15 \mathrm{~F}$ (à peu près 50 centimes), il est vrai pour un nombre nettement plus réduit de pages. En l'occurrence, le but de la collection n'était pas seulement de maximiser les profits de l'éditeur ou de permettre aux non-abonnés (soit la très grande majorité du lectorat) de disposer de l'ensemble du récit aimé au cas où on aurait raté un numéro en kiosque. En fait, l'objectif principal du lancement de la collection était tout autre : faire événement, et à travers cette intervention, mettre en place des «stratégies publicitaires centrées sur les publics cibles", voire jouer un "rôle dans la construction des sociétés contemporaines » (Rivalan Guégo et Nicoli 2014, 11 et 15).

En premier lieu, les Éditions Mondiales cherchaient à mettre en valeur une forme de récit tenu en piètre estime et n'ayant jamais fait l'objet d'une publication sous forme de « livre » (à titre de comparaison : en bande dessinée aussi, la reprise en album est restée rare jusque vers la fin des années cinquante, voir Boillat et Revaz 2013). Le prestige attaché à l'objet-livre était censé rejaillir sur l'éditeur, méprisé par bon nombre de ses 
confrères, mais aussi sur les lecteurs, culturellement et économiquement tenus à distance de l'achat de livres (rappelons que le « Livre de poche » ne surgit qu'en 1953).

Deuxièmement, la création de cette collection de romans dessinés relevait aussi d'impératifs commerciaux de grande urgence. Lancé un an après Grand Hôtel, dont au début il n'était guère qu'une traduction hâtive, Nous Deux arrivait en kiosque au même moment où l'Italie, puis rapidement la France voyaient arriver le roman-photo. Grâce au triomphe de leurs superbes romans dessinés, tant Grand Hôtel que Nous Deux ont pu ignorer pendant quelque temps la concurrence du nouveau venu, jusqu'au moment où le roman-photo commence vraiment à grignoter des parts de marché. Ce n'est que le 9 août 1950 que Nous Deux présentera son premier roman-photo, inévitablement traduit de l'italien Grand Hôtel: la manœuvre lui permettra de prendre juste à temps le train en marche et de pérenniser sa position hégémonique dans la presse du cœur.

Sans doute le combat en faveur du roman dessiné était-il perdu d'avance, l'esprit du temps ayant vite fait le choix du roman-photo. Il n'empêche que la collection des "romans dessinés de Nous Deux » était une pièce clé dans la stratégie commerciale du patron des Éditions Mondiales, Cino Del Duca, qui même après l'entrée du roman-photo dans Nous Deux, continuait à investir dans le roman dessiné jusque dans la seconde moitié des années 50 .

La collection de livres y jouait un rôle d'autant plus décisif qu'on assistait rapidement à un changement d'orientation: au lieu de reprendre des matériaux déjà parus, la collection s'est tournée aussi vers les «inédits ». ${ }^{2}$ Bref, le genre même du roman dessiné, puis l'existence d'une collection de livres et enfin son ouverture à des œuvres originales sont tous pur signe de cette distinction culturelle, celle du livre (avec majuscule), qui, d'après Del Duca, faisait défaut à ses concurrents, limités à un genre moins «artistique " (le roman-photo, le dessin étant toujours jugé plus noble que l'image mécanique de la photographie), à un mode de publication "populaire " (le feuilleton) et enfin à un support moins prestigieux (les magazines vendus en kiosque, soit hors librairie). ${ }^{3}$

15 Mais il y a visiblement erreur sur le genre. Le plaisir du roman dessiné comme du roman-photo vient en grande partie de son existence feuilletonesque, du suspense qu'elle entraîne, de la pulsion narrative qui est la sienne, des va-et-vient entre l'œuvre et les autres sections du magazine où paraissent ces romans visuels. Menacé, et bientôt totalement défait, par le roman-photo, le roman dessiné survit mal à sa promotion de roman-feuilleton en roman complet. Quant au challenger victorieux, le roman-photo, il n'a guère besoin de se risquer à sauter le pas du magazine au livre.

16 La situation sera tout autre quand s'ouvre l'ère du ciné-roman-photo (Baetens 2019), sous-genre de roman-photo où la notion de feuilleton n'a plus guère de sens, puisque les magazines de ciné-romans-photos ne sortent qu'à la fin de l'exploitation des films dans les grandes salles (et souvent même après leurs deuxième et troisième vies dans les salles de quartier et de province). Or les magazines du ciné-roman-photo, qui ne publient en principe que des récits complets (à quoi bon découper en épisodes ce qui est de toutes façons déjà connu du public ?), sont gouvernés intégralement par la logique de collection. Les ciné-romans-photos ne paraissent pas seulement dans tel ou tel magazine, comme c'est le cas des romans-photos, ils prennent également place tout de suite dans des collections spécialisées, qui sont autant de filiales d'une maison-mère liée à l'univers du roman-photo. Cette politique de la double enseigne, combinant magazine et collection, s'observe nettement dans le cas de Nous Deux, acteur clé du 
segment ciné-photo-romanesque et offrant à ses lecteurs le choix entre : Nous Deux Film, Nous Deux Film Moderne, Nous Deux Aventures Action, Nous Deux Roman-Film Étoile, Nous Deux Roman-Film Série d'or, Nous Deux Roman-Film Vedette (pour ne rien dire des autres magazines du même éditeur, eux aussi très actifs dans le filon porteur, du moins dans la décennie 1955-1965, du ciné-roman-photo).

Bref, roman-photo et ciné-roman-photo sont l'un et l'autre des produits hyper-sériels, mais seul le dernier a été en mesure d'accéder à la collection. La situation ne changera que dans les années soixante, au moment de la rencontre entre un nouveau support, les " petits formats » (Capart 2015), et de nouveaux genres, le mélodrame étant chassé par la science-fiction, l'horreur et la pornographie (ou une combinaison de plusieurs de ces catégories, comme dans le célèbre Satani $k^{4}$ ). Le roman-photo traditionnel, dont l'âge d'or s'éteint avec l'arrivée de la télévision dans les foyers, ne fait pour ainsi dire jamais l'objet d'une publication indépendante. Il reste confiné à l'intérieur d'un magazine à la table des matières bigarrée. Tel manque d'autonomie l'empêche de se construire en collections, sauf au deuxième degré : un roman-photo de Nous Deux n'est pas un romanphoto de la collection Nous Deux mais un roman-photo publié dans le magazine du même nom et qui respecte et reflète le style, les thèmes, les valeurs du magazine.

\section{Une autre manière de faire collection : hors série?}

Dans le roman-photo, une dimension essentielle de la sérialité semble donc faire défaut : la collection. Tel est du moins le cas du roman-photo traditionnel. Il en allait autrement, on vient de le voir, dans les collections ultérieures comme Satanik. Cette nouvelle catégorie de romans-photos change toutefois de genre, dans la double acception du terme : on passe du récit sentimental au récit policier en même temps que le public visé cesse d'être de sexe féminin (dans Satanik la femme est vraiment objet, si ce n'est chose -ce qui ne signifie pas nécessairement que seuls les hommes prenaient plaisir à cette lecture). Quant au roman-photo " art et essai », brillamment illustré dans les années 1980 par un livre comme Droit de regards (Plissart et Derrida, 2010), il a lui aussi pâti du même problème, son promoteur principal, les éditions de Minuit, n'étant jamais parvenu à créer un effet de collection autour d'une série d'œuvres qui aux yeux du public restaient dépareillées.

Or la collection n'est pas le monopole de l'éditeur. Elle peut résulter aussi d'un geste du public, qui constitue ses propres collections, pour les divulguer ensuite de manière de plus large. ${ }^{5}$ Il arrive même que ces collections d'amateur finissent par prendre la place des collections établies ou de s'y substituer en cas d'absence de toute collection. Pareille démarche peut soit redoubler le geste de l'éditeur (s'agissant du roman-photo, c'est le cas de tous ceux qui gardent les magazines pour les relier en volumes, quitte à en détacher les seules pages photo-romanesques), soit obtempérer à d'autres critères (par exemple lorsqu'on tente de réunir les publications en fonction d'éléments thématiques, formels ou de casting : tous les romans-photos situés en Écosse, tous les romans-photos avec Johnny, tous les romans-photos colorisés publiés entre 1960 et 1964, etc. $\left.{ }^{6}\right)$. Le roman-photo se prête admirablement à ce geste de collection sans doute moins canonique mais pour le coup très " actif ». Une telle manière de faire suscite de nouveaux ensembles au lieu de " passivement» (sic) se contenter de refaire ce qui avait été fait, mais pas forcément maintenu ou entretenu par l'éditeur, évitant par exemple la dispersion à moyen ou long terme de ce qui faisait série au moment de sa sortie. 
20 Dans le roman-photo le vide institutionnel de la collection est net. Les archives des nombreuses structures éditoriales qui en ont publié ont souvent disparu. Et quand elles existent encore, elles sont souvent peu accessibles et mal classées (oui, les boîtes à chaussures subsistent vaillamment!). Or chaque archive retrouvée s'avère sans exception être une véritable caverne d'Ali Baba, comme l'ont montré à profusion trois grandes expositions récentes autour du genre. D'abord Roman-photo au MUCEM de Marseille (du 13 décembre 2017 au 23 avril 2018), qui a exploré les images originales des éditions italiennes Mondadori. ${ }^{7}$ Ensuite Sul set. Fotoromanzi, genere e moda nell'archivio di Federico Vender à Trento (du 8 septembre 2017 au 7 janvier 2018), qui a présenté des documents du studio Vender, spécialisé dans le portrait en noir et blanc, un peu comme Harcourt en France, mais fort présent aussi dans un certain type de roman-photo, celui des grandes adaptations littéraires de la revue Luna-Park. ${ }^{8}$ Enfin Fotoromanzo e poi... à Reggio Emilia (du 20 avril au 17 juin 2018), exposition construite autour d'une œuvre " exhumée ", car publiée sous pseudonyme, de Cesare Zavattini, grand scénariste du néo-réalisme italien. ${ }^{9}$ Chacune de ces expositions a distingué dans le monde sériel du roman-photo un certain nombre de pistes capables de faire surgir des jonctions qui chemin faisant ont pris la forme de véritables collections : de genre (comme le romanphoto historique à Trento), de style (comme le roman-photo érotique à Marseille), de thème (comme le féminisme à Reggio).

21 Mais même lorsque la production photo-romanesque est prise en charge par des institutions muséales ou autres et devient consultable en archive, les lacunes sont criantes. Le dépouillement effectué par les bibliothèques nationales par exemple, outil essentiel de n'importe quel chercheur, est souvent rudimentaire. Les romans-photos inclus dans tel ou tel numéro de magazine restent invisibles dans les fichiers et répertoires, qui ne descendent pas jusqu'aux détails des sommaires. D'où l'intérêt d'un projet comme «Photo-Lit ", qui dans le cadre d'un travail de numérisation du romanphoto belge "caché » dans les dépôts de la Librairie Royale (c'est-à-dire Nationale) a mis au point des protocoles de description bibliographique "sur mesure", tenant compte des particularités du genre photo-romanesque. La notion d' "auteur » y est particulièrement épineuse, faute d'indications paratextuelles, car il n'est pas rare que les œuvres soient totalement anonymes, mais aussi à cause du caractère collectif de la production.$^{10}$

22 Répétons-le : les absences de collection, à quelque niveau et de quelque forme que ce soit, sont légion dans le domaine du roman-photo, mais elles sont tout sauf une malédiction. Le déficit de structures fortes facilite en effet l'avènement de nouveaux modes d'organisation, plus appropriés aux formes contemporaines de la lecture, qui tend vers l'appropriation et la transformation des objets d'origine. La révolution numérique accélère ces phénomènes, par exemple en stimulant la création d'archives qu'Abigail De Kosnik (2016) qualifie de «sauvages », « rebelles » ou " pirates » (si l'on m'autorise à traduire ainsi l'adjectif anglais « rogue »). Montées par des fans dans le contexte plus large de la culture participative, ces archives modifient le corpus qu'elles traitent autant qu'elles le conservent. Elles sont structurées en fonction des préférences subjectives de l'archiviste, loin de tout respect des règles de l'art archivistique (elles rejettent catégoriquement toute limitation imposée par les régulations actuelles du copyright par exemple). Elles procèdent à des regroupements inédits, qui résultent parfois en des combinaisons non seulement hors collection mais littéralement hors série (et non pas seulement «hors-série", avec trait d'union, 
formule qui ne suppose aucune vraie rupture avec la série sur laquelle se greffe le nouvel élément). Elles brouillent les frontières entre œuvre et commentaire. Enfin, elles aspirent souvent à se faire productrices, encourageant leurs utilisateurs à ajouter leurs propres matériaux, voire à créer de nouvelles œuvres pour remédier aux lacunes des matériaux disponibles.

Ce travail d'archiviste "rebelle » peut facilement être prolongé, refait, défait par le public, désormais capable d'accomplir les gestes de celle ou de celui dont il juge le travail. ${ }^{11}$ N'importe quel utilisateur minimalement instruit des possibilités du net peut aujourd'hui créer - ou du moins tenter de le faire - sa propre collection (bibliothèque, discothèque, filmothèque), sans être obligé de respecter la structure interne aussi bien qu'externe des objets qu'il agrège : d'une part, les œuvres sont démontées : fragmentées, divisées, sectionnées; d'autres part, elles sont remontées: remembrées sans tenir compte des séries ou des collections dont elles proviennent (voir Bonnard 2018, qui se penche sur les catalogues de films sur la Toile).

Ces dernières observations nous éloignent un peu du roman-photo, peut-être, mais elles nous ramènent aussi vers la question essentielle de ces pages, à savoir la tension productrice entre série et collection. Le travail en série implique forcément la notion de collection : ce qui se crée en série a pour horizon de faire collection. Mais série et collection sont loin de toujours coïncider. Le roman-photo est une pratique qui semble faire l'économie de la collection, mais celle-ci en est tout sauf absente. D'abord implicitement, puisqu'au niveau supérieur du magazine, la collection est une donnée capitale. Ensuite de manière plus explicite, mais différente, par exemple quand les lecteurs s'emparent du corpus pour convertir la série en collection. Ce nouveau type de collection n'est pas seulement quelque chose qui s'ajoute à la série, il est aussi une force qui la transforme, l'ignore, la casse, la réinvente, pour instituer en quelque sorte de série "bis ", à la fois différente de la série originale et radicalement ouverte à de nouveaux agencements. Pareille leçon n'est pas nécessairement sensationnelle, mais elle a au moins l'avantage de dire clairement à quel point le travail en série, loin des idées de répétition mécanique qu'il peut véhiculer, est aussi une pratique fondamentalement créatrice.

\section{BIBLIOGRAPHIE}

Antonioni, Michelangelo (1949). L'amorosa menzogna. Rome : Filmus/Edizioni Fortuna. En ligne : https://www.youtube.com/watch?v=1oMv4uIVNIs (consulté le 28 mai 2020).

Antonutti, Isabelle (2013). Cino Del Duca: De Tarzan à Nous Deux; Itinéraire d'un patron de presse. Rennes: Presses Universitaires de Rennes.

Artiaga, Loïc, et Matthieu Letourneux (2013). Fantômas! Biographie d'un criminel imaginaire. Paris: Les Prairies Ordinaires.

Baetens, Jan (2017). Pour le roman-photo, édition revue et élargie. Bruxelles : Les Impressions Nouvelles. 
--- (2018) « Les épisodes du roman dessiné », in Cahiers de narratologie 34. En ligne : https:// journals.openedition.org/narratologie/8866 (consulté le 29 mai 2020).

--- (2019). The Film Photonovel. A Cultural History of Forgotten Adaptations. Austin : Texas University Press.

Boillat, Alain, et Françoise Revaz (2013). La BD avant l'album. Exposition du GREBD à l'Hôtel de ville de Lausanne. En ligne : https://wp.unil.ch/grebd/evenements/expositions-du-grebd/ (consulté le 29 mai 2020).

Bonifazio, Paola (2017). « Political Photoromances: The Italian Communist Party, the Catholic Press, and the Battle for Women's Heart. »Italian Studies 72:4, 393-413.

Bonnard, Martin (2018). «(Re)monter le cinéma sur le weg”. CInémas 28: 2-3, 93-109. En ligne: https://www.erudit.org/en/journals/cine/1900-v1-n1-cine05122/1067495ar/abstract/ (consulté le 29 mai 2020).

Bravo, Ana (2003). Il fotoromanzo. Bologne : Il Mulino.

Capart, Philippe, dir. (2015). « Les patrons de la bande dessinée », La Crypte tonique 12.

Collectiana (2020). Collectiana. Fondation pour l'étude et le développement des collections d'art et de culture. En ligne : https://www.collectiana.org/

De Kosnik, Abigail (2016). Rogue Archives: Digital Cultural Memory and Media. Cambridge, Mass.: MIT Press.

Deschamps, Frédérique, et Marie-Charlotte Calafat, dir. (2017). Roman-photo. Paris : éd. Textuel, et Marseille : MUCEM.

Faber, Dominique, Marion Minuit et Bruno Takodjerad (2012). Nous Deux présente : La Saga du roman-photo. Paris : éd. Jean-Claude Gawsewitch.

Genette, Gérard (1982). Palimpsestes. La littérature au second degré. Paris: Seuil.

Giet, Sylvette (1998). Apprendre la langue du cœur. Nous Deux 1947-1997. Leuven : éd. Peeters, et Paris : éd. Vrin.

Le Péron, Serge (2020). « Macadam à deux voies ", in Frédéric Sojcher et Serge Le Péron, dir., Cinéma à l'université. Le Regard et le geste. Bruxelles : Les Impressions Nouvelles, 44-57.

Malatesta, Katia (2017). Sul Set. Fotoromanzi, genere e moda nell'archivo di Federico Vender. Trento: Provincia autonoma di Trento.

Pinchon, Pierre (2018). Godard contrebandes. 1960-1968. Montreuil : éd. Matière.

Plissart, Marie-Françoise (2010 [1985]). Droit de regards. Avec une lecture de Jacques Derrida. Bruxelles : Les Impressions Nouvelles.

Rivalan Guégo, Christine, et Miriam Nicoli, dir. (2014). La Collection. Essor et affirmation d'un objet éditorial. Rennes : Presses Universitaires de Rennes.

Sullerot, Évelyne (1963). La presse féminine. Paris, Armand Colin.

\section{NOTES}

1. Rappelons que dans la taxinomie des relations textuelles de Gérard Genette (1982), la relation hypertextuelle désigne toute relation unissant un texte $\mathrm{B}$ (hypertexte) à un texte antérieur $\mathrm{A}$ (hypotexte) sur lequel il se greffe d'une manière qui n'est pas celle du commentaire. 
2. Pour une analyse des complications narratives de ce changement, qui oblige les auteurs à repenser le découpage du récit en épisode, voir Baetens (2018).

3. Plus tard, Del Duca se lancera aussi dans la production cinématographique (il a cofinancé entre autres des films comme L'Avventura de Michelangelo Antonioni) et le mécénat littéraire (placé aujourd'hui sous les auspices de l'Institut de France, le "Prix Mondial" de la Fondation Del Duca, doté de 200.000 euros, est souvent considéré comme le prix Nobel français).

4. Satanik était une série de romans-photos traduits de l'italien, dont 19 numéros ont été publiés en France en 1966-1967, avant son interdiction par la censure pour cause de violence extrême et d'érotisme outré (les amateurs du genre savent que l'original italien s'intitulait Killing, le Satanik italien étant quant à lui une série de bandes dessinées à protagoniste féminin, traduite en français sous le nom de Diabolik). L'interdiction de Satanik a donné lieu à la multiplication de toutes sortes de clones, moins portés sur la violence que sur l'érotisme. La collection particulière de l'auteur de cet article, qui est sans doute loin d'être complète, comprend: Bluff, Casino, Cristal, Fièvres, Halt, Pièges, Rafles, Ravages, Rondes (éditions Brandt), Duel, Fugue, Hold-up, La Mafia, La Main noire, Racket, Razzia, Trafics (éditions de Poche), Bis, Brio, Duo, Evasions, Favorites, L'Ombre, Paréo, Roc (éditions Janvier). Pour une étude historique du personnage de Satanik, avatar moderne de Fantômas, voir Artiaga et Letourneux (2013).

5. Voir le site portail Collectiana.org, consacré aux collections privées : Collectiana 2020.

6. Autant de critères qui se croisent et se combinent à volonté, ce qui laisse aussi rêver de la face cachée du genre: quid d'un roman-photo colorisé, avec Johnny, situé en Écosse et publié juste avant le premier 33 tours de Jean-Philippe Smet?

7. Pour plus d'informations, voir le site de l'exposition: http://www.mucem.org/programme/ exposition-et-temps-forts/roman-photo, et le catalogue (Deschamps et Calafat 2017). C'est dans le sillage de cet événement qu'a pu sortir la «collection » des ciné-romans-photos de Jean-Luc Godard (Pinchon 2018). Signalons aussi que cette exposition a été reprise au musée de la photographie de Charleroi, avec un volet belge sans doute moins connu du grand public.

8. Voir https://www.cultura.trentino.it/Approfondimenti/Sul-set.-Fotoromanzi-genere-e-modanell-archivio-di-Federico-Vender et le catalogue (Malatesta 2017).

9. Voir: https://www.fotografiaeuropea.it/fe2018/mostra/fotoromanzo/ (il n'existe malheureusement pas de catalogue de cette exposition). On pouvait voir à Reggio le contrat original avec l'éditeur, dans lequel Zavattini avait fait stipuler que le voile sur la véritable identité du scénariste ne pouvait en aucun cas être levé.

10. Pour plus de détails, voir le site du projet (une collaboration de la Bibliothèque Royale et des universités de Leuven et de Liège : https://www.photolit-brain.com/, et le site portail donnant accès aux œuvres numérisées (plus de six cents romans-photos): https://belgica.kbr.be/ BELGICA/photonovels.aspx

11. Pour une comparaison avec le domaine du cinéma, notamment avec l'apparition d'un spectateur du « troisième » type, capable de devenir réalisateur à son tour, voir Le Péron 2020.

\section{RÉSUMÉS}

Série et collection sont des amis, mais de faux amis. De l'une à l'autre, les correspondances sont nombreuses, mais pas au point d'en faire de vrais synonymes. Objet sériel en même temps qu'objet de collection, le roman-photo offre une belle occasion de réfléchir aux ressemblances et dissymétries des deux concepts et des deux pratiques. Dans le contexte du roman-photo, la 
production de la série et celle de la collection obéissent à des logiques différentes. Autant le médium du roman-photo obéit à la logique sérielle et ses plus lourdes contraintes (en l'occurrence: conception et réalisation de l'œuvre sous forme de feuilleton, cadence de production élevée, conformité absolue aux règles narratives et stylistiques du mélodrame hérité des romans du 19e siècle) autant il se conjugue mal avec le principe de la collection, que certains éditeurs essaient de mettre en avant comme signe de distinction, sans trop de succès toutefois, dans le cas de l'ancêtre du roman-photo, le roman dessiné. Le déficit de la collection et le goût de la distinction se retrouveront dans une phase ultérieure du roman-photo, quand ce dernier essaiera de produire des « hors-série ».

INDEX

Mots-clés : roman-photo, roman dessiné, collection, sérialité, mélodrame

\section{AUTEUR}

\section{JAN BAETENS}

Jan Baetens est professeur d'études culturelles à l'université de Leuven, où il s'intéresse notamment aux rapports entre texte et image dans des genres hybrides tels que la bande dessinée ou le roman-photo. Quelques publications récentes sont: Adaptation et bande dessinée (Les Impressions Nouvelles, 2020) et Une fille comme toi (JBE éditions, 2020), un "remix" d'une collection de ciné-romans-photos à partir du scénario imaginaire d'un film typique de la Nouvelle Vague.

jan.baetens@kuleuven.be 